\title{
Technical Approaches to Computer-Assisted Orthopedic Surgery
}

\author{
Frank Langlotz, Lutz-Peter Nolte ${ }^{1}$
}

\begin{abstract}
Surgical navigation systems and medical robotic devices are increasingly being used during trauma and orthopedic surgery. This article tries to present the underlying technology of these devices and to describe different approaches to the various aspects of the methods. To structure the variety of available products and presented research modules, a new categorization for these approaches is proposed. Examples of pre- or intraoperative imaging modalities, of trackers for navigation systems, of different surgical robots, and of methods for registration as well as referencing are discussed. Many applications that have been realized for numerous surgical procedures will be presented and their advantages, disadvantages, and possible implications will be elucidated.
\end{abstract}

\section{Key Words}

Navigation · Robotics · Registration · Categories of CAOS

Eur J Trauma 2004;30:1-11

DOI 10.1007/s00068-004-1374-0

\section{Introduction}

For about 1 decade, novel medical robotics as well as computer-assisted surgery tools and instruments have been continuously introduced into orthopedic and traumatologic surgery rooms throughout the world. Meanwhile, these systems have been applied to a considerably large number of surgical interventions, and they might even be about to become state-of-the-art for certain applications. Over the years, different concepts and

\footnotetext{
'Institute for Surgical Technology and Biomechanics, M.E. Müller Research Center for Orthopedic Surgery, University of Bern, Switzerland.
}

Received: October 1, 2003; accepted: November 15, 2003. techniques have been developed, realized, and evaluated. Some of them proved to be successful, while others appeared to be dead-end roads and have consequently been abandoned in the meantime.

This article tries to structure those approaches and sorts them into a proposed scheme. During this categorization, the described methods are elucidated from a technical point of view, and clinical examples are presented. Although focusing mainly on the field of traumatology, techniques that were first or only employed in other domains such as arthroplasty or the treatment of the degenerated spine will be presented within their respective fields of application.

\section{Categorization of CAOS (Computer-Assisted Orthopedic Surgery) Systems}

Both traumatology and orthopedic surgery aim at the treatment of bony structures or tissue directly connected to bones that are usually located deep inside the human body. Surgical steps such as the placement of an implant component, the reduction of a fracture, or the cutting or drilling of bone should ideally be carried out as precisely as possible. Not only will optimal precision improve the postoperative biomechanical performance of the treatment [1,2], but also guarantee that the probability of intra- and postoperative complications is minimized [3]. A large number of mechanical guides have been developed for various applications in orthopedics and traumatology. While many of them surely help improve surgical precision, their general benefit has been questioned (e.g., [4]). Surgical skills and expertise are definitely the premier methods to achieve a positive operative outcome. However, limited visibility makes it often difficult to realize the intended procedure as accurately as desired. Large surgical exposures are certainly 
inappropriate ways to improve visibility, due to the associated tissue damage. Moreover, challenging new techniques of minimally invasive treatment make it more and more important to gain feedback about the action that takes place subcutaneously. Just as laparoscopy and arthroscopy have introduced video transmission to present recorded images of the situs on a video monitor, CAOS mimics surgical action in real time using a virtual scene of the situs presented on a computer monitor [5]. This technique was initially developed for frameless intracranial interventions, and after further refinement can now cover various procedures in orthopedics and traumatology. Another method to potentially improve the outcome of bone surgery is the employment of surgical robots. Being successful in industrial production for many years, their precision and their resistance against tremor and fatigue have been advocated for different applications in traumatology and orthopedics.

Although the different applications use numerous technical methods to realize individual aspects of a navigation system or surgical robot, the conceptual design of each of these devices is similar, and an associated categorization has been proposed by Bowersox et al. [6]. They define three major components involved in the clinical application of surgical navigation: a therapeutic object (the target of treatment), a virtual object (its counterpart in the navigation and planning computer), and a so-called navigator that links both objects. For reasons of simplicity, the term "CAOS system" will be used within this article to refer to both navigation systems and robotic devices. Although Bowersox's global categorization is still valid for the large diversity of today's CAOS systems, a finer classification (Figure 1) appears more suitable to respect the various techniques that have been realized for certain aspects of CAOS systems.

The central element of each CAOS system is the so-called navigator. It establishes a global, threedimensional coordinate system and thus enables the transmission of positional information between the virtual object (VO) and the therapeutic object (TO). For robotic devices, the robot itself plays the role of the navigator, while during surgical navigation, the tracker serves as navigator. The VO and TO are mathematically linked to the navigator by registration (VO) and referencing (TO). The $\mathrm{VO}$ represents an image of those parts of the anatomy that are operated on with the help of the CAOS system. Examples for each of these elements will be presented and discussed in the following sections.

\section{Virtual Object}

The purpose of the VO in each CAOS system is to provide a realistic representation of the bony structures involved in a surgical intervention. The image data is visualized on a computer monitor and provides the framework in which robotically assisted procedures can be planned. Navigation systems use these images for preoperative planning as well. In addition, they serve as the intraoperative "background" onto which the measured position of a surgical instrument is projected, as exemplified in Figure 2.

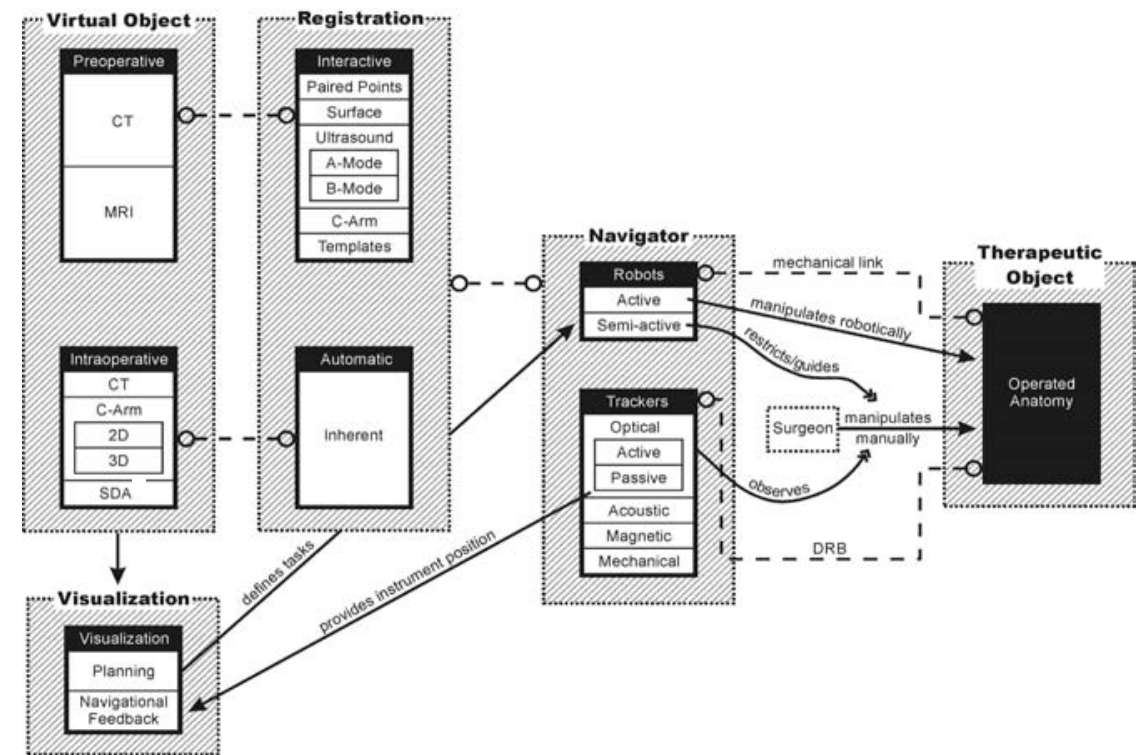

Figure 1. Diagram of the categorization of CAOS (computer-assisted orthopedic surgery) systems. The three main elements of each CAOS system - virtual object, navigator, and therapeutic object - are connected by registration and referencing. Virtual objects can be acquired preoperatively, which requires interactive registration, or intraoperatively, usually resulting in an automated, inherent registration. The visualization of the image data allows for intervention planning and provides the framework in which navigational feedback is provided. Surgical robots either carry out certain steps of an operation or restrict or guide the surgeon in doing so. Observing the surgeon's actions enables a navigation system to visualize this action in real time. The connection between the navigator and the therapeutic object is established by a mechanical linkage or a dynamic reference base (DRB). CT: computed tomography; MRI: magnetic resonance imaging; SDA surgeon-defined anatomy. 
VOs may be acquired at two points in time: either preoperatively or intraoperatively. About 1 decade ago, the first CAOS systems that were presented were based on preoperatively acquired computed tomography (CT) scans. The advantage of this modality is that it provides excellent bone-soft tissue contrast. Moreover, the acquired images are geometrically undistorted and, thus, no sophisticated calibration needs to be applied. These advantages make CTs superior to magnetic resonance imaging (MRI) as preoperative VOs, although the latter method has clear advantages regarding radiation exposure to the patient. Some efforts have been made to overcome the MRI-related difficulties [7, 8]; however, up to now, CT remains the method of choice of preoperative imaging for CAOS applications. Another drawback of preoperative VOs led to the introduction of intraoperative imaging modalities. The bony morphology may change between the time of image acquisition and the actual surgical procedure. As a consequence, the VO may not necessarily correspond to the $\mathrm{TO}$, leading to unpredictable inaccuracies during navigation or robotic procedures. This effect can be particularly adverse for traumatology in the presence of unstable fractures. To overcome this problem in the field of surgical navigation, the use of intraoperative CT scanning has been proposed [9], but the infrastructural changes that are required for the realization of this approach are tremendous, often requiring considerable

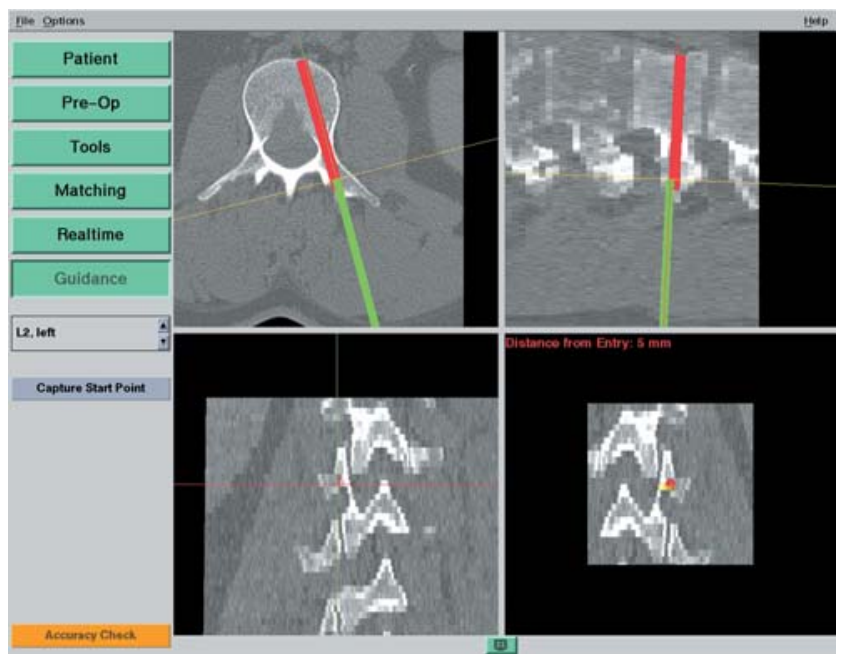

Figure 2. Example of navigational feedback. In this case of CT-based navigation during pedicle screw placement, the optimal location for the screw in L2 has been planned preoperatively (red line). The current position and orientation of the instrument used to prepare the screw canal is overlaid as a green line, facilitating precise alignment of the instrument with the plan. reconstruction of a hospital's facilities [10]. An alternative is the use of established intraoperative imaging modalities. Several research groups developed navigation systems based on fluoroscopic images [11, 12]. The fluoroscope is a well-established device during orthopedic and trauma treatment and could therefore be integrated into CAOS systems more easily than intraoperative CT. In contrast to CT though, the images generated with a fluoroscope are usually distorted, which is caused by a number of factors. To use fluoroscopic images as VOs therefore requires calibration of the fluoroscope, involving the attachment of marker grids to the image intensifier and the tracking of its position and orientation with the navigator during image acquisition [11, 12]. The resulting real-time visual feedback provided by the navigation system (see Figure 3 ) is similar to the use of the fluoroscope in constant mode. This technique is therefore also known as "virtual fluoroscopy" [13]. Although only two-dimensional projections are available and the images usually lack contrast when compared to preoperative CT, the advantages of fluoroscopy-based navigation preponderate for a number of clinical applications.

Recently, a novel imaging device has been developed [14] that enables the intraoperative generation of three-dimensional, fluoroscopic image data. It consists of a motorized, isocentric $\mathrm{C}$-arm that acquires series of 50-100 two-dimensional projections and reconstructs

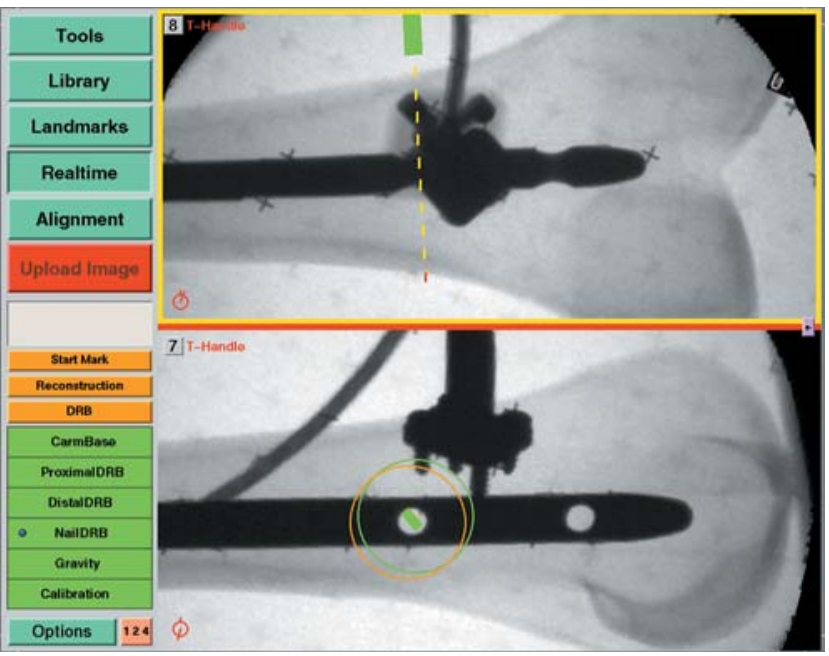

Figure 3. Fluoroscopy-based navigation. This screenshot shows the distal locking of an intramedullary nail in a plastic bone model. Two different fluoroscopic images are displayed simultaneously with the current orientation and location of a surgical instrument overlaid as colored graphics. 
from them $13 \times 13 \times 13 \mathrm{~cm}^{3}$ volumetric datasets which are comparable to CT scans. Initially advocated primarily for surgery at the extremities, this "fluoro-CT" has been adopted for use with a navigation system and has already been applied to several anatomic areas (see Clinical Fields of Application). As a major advantage, the device combines the availability of three-dimensional imaging with intraoperative data acquisition.

A last category of navigation systems functions without any radiologic images as VOs. Instead, the tracking capabilities of the system are used to acquire a graphic representation of the patient's anatomy by intraoperative digitization. Using any tracked instrument, the spatial location of anatomic landmarks can be recorded. Combining the obtained points into lines and surfaces will generate, step by step, an abstract model of the geometry. Since this model is generated by the operator, the procedure is known as "surgeon-defined anatomy" (SDA). The technique is particularly useful when soft tissue structures such as ligaments or cartilage boundaries are to be considered that are difficult to identify on CTs or fluoroscopic images. Moreover, some locations can be acquired without direct access of a digitizing instrument. For instance, the center of the femoral head, which is an important landmark during total knee replacement, can be reconstructed from recorded passive rotation of the leg around the acetabulum. However, the generated images are often rather abstract and not easy to interpret as exemplified in Figure 4. Sati et al. [15] suggested underlaying a preopera-

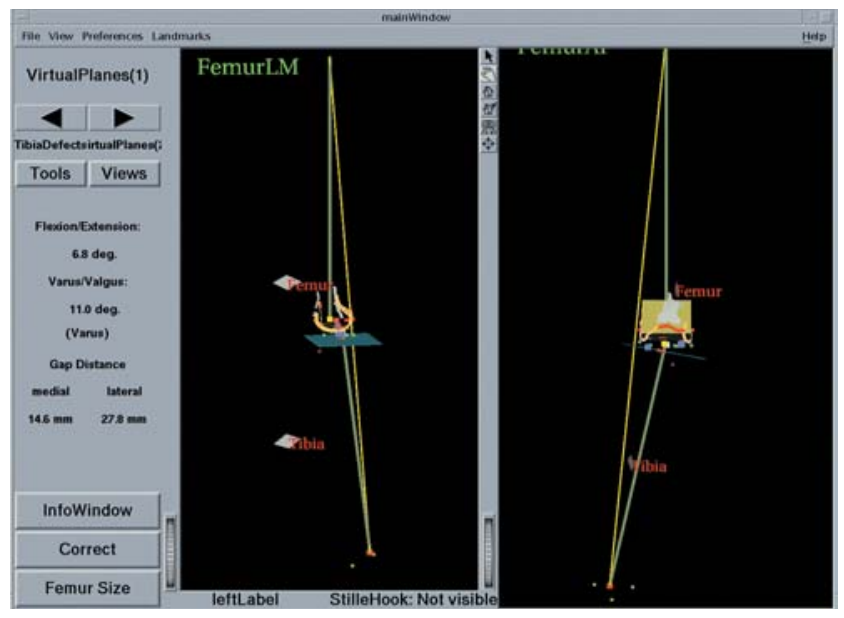

Figure 4. Navigation using the surgeon-defined anatomy (SDA) approach. This virtual model of a patient's knee is generated intraoperatively by digitizing relevant structures. Although a very abstract representation, it provides sufficient information to enable navigated placement of a total knee endoprosthesis. tive X-ray to facilitate orientation, but the precise matching of the two image spaces turned out to be difficult. An alternative concept is provided by the so-called bone morphing [16, 17]. This process uses a database of generic, three-dimensional, statistical computer models of bones and a set of specific points that are acquired with the SDA technique. Analyzing the recorded data lets the system select the bone model from the data pool that best matches the patient's morphology. A special morphing algorithm would then deform the selected model three-dimensionally until it fits the acquired points as perfectly as possible. As a result, a realistic virtual model of the operated structure can be presented and used as a $\mathrm{VO}$ without any conventional image acquisition (see Figure 5).

\section{Registration}

Position data that is used intraoperatively to display the current tool location (navigation system) or to perform actions according to a preoperatively planned step (robot) is expressed in the local coordinate system of the VO. In general, this coordinate system differs from the one in which the navigator operates intraoperatively. In order to bridge this gap, the mathematical relationship between both spaces needs to be determined. In the case of preoperative images serving as VOs, this step is performed interactively by the surgeon during registration, also known as matching. A wide variety of different approaches have been developed and realized following numerous methodologies.

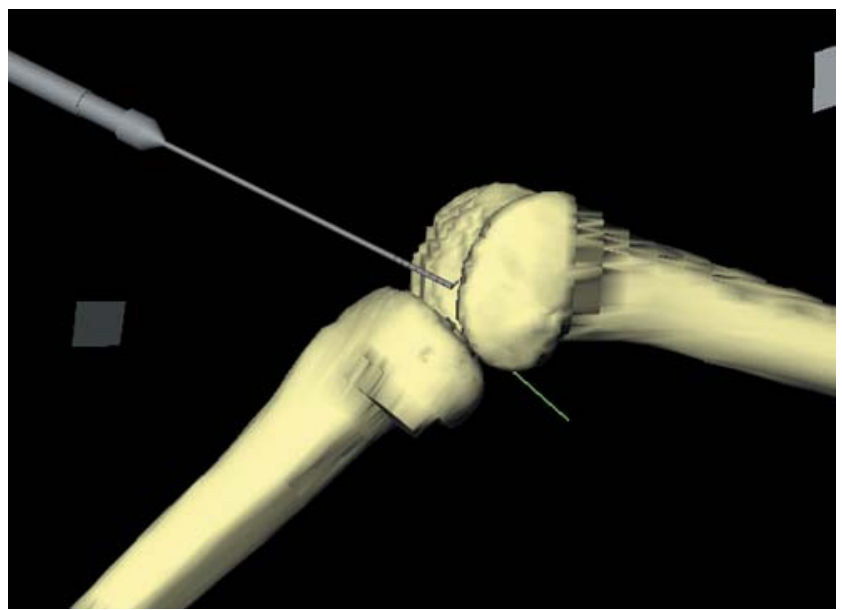

Figure 5. Bone morphing. The points that were digitized by means of the surgeon-defined anatomy (SDA) approach are used to deform a statistical knee model into the actual patient's knee. This technique provides a very realistic, individualized representation of the operated anatomy without any conventional imaging modality. 
The first CAOS systems implemented a featurebased registration [18]. The surgeon teaches the system the aforementioned coordinate transformation by presenting it the correspondence for several representatives. The technically simplest method of this category is the so-called paired-points registration. Pairs of distinct points are defined preoperatively in the $\mathrm{VO}$ and intraoperatively in the TO. The former set of points is usually identified using the computer mouse to mark the desired location within the image data. For intraoperative acquisition, a probe is used. In the case of a navigation system, it is tracked by the navigator, and for robotic surgery, it is mounted onto the robot's actuator, which the surgeon then passively guides to the location to be recorded [19]. Once both point sets are available, the transformation that links the underlying coordinate systems can be derived. It is obvious that this procedure is highly interactive during both the preoperative definition of registration points and the intraoperative acquisition of their counterparts. Consequently, this step is error-prone, in particular because a good registration result and, thus, accurate performance of the CAOS system strongly depends on the optimized selection of the registration points and the exact identification of the pairs. To improve the accuracy of this step, alternative and complementing techniques have been proposed. Probably most obvious is the implantation of artificial objects to create easily and precisely identifiable spots for paired-points registration. Percutaneous markers [20], pins [19], screws [21], or complex marker carriers [22] have been suggested. However, these methods require the artificial markers to be represented in the preoperative image as well, thus necessitating implantation prior to CT scanning during an additional intervention. Although this is usually done under local anesthesia, the extra operation incurs further costs, not to mention the associated discomfort for the patient [23]. Consequently, none of these methods have gained wide clinical acceptance.

Radermacher et al. [24] presented a different approach to overcome the registration problem. They extracted distinctive areas of the bony surface from the preoperative CT data. Using rapid-prototyping techniques, they then fabricated a negative mold. Intraoperatively, this item would be placed onto the corresponding bone area, where it would fit at one spot only thanks to it characteristic shape. During the manufacturing of this template, jigs for drilling or cutting can be incorporated that automatically align a surgical instrument in a desired orientation with the bone. For example, a tube at the intended axis of a pedicle screw can serve as a drill sleeve enabling the exact realization of a planned screw trajectory. Although this concept is still applied [25], it never became widely used, mainly due to the associated logistic efforts related to the rapid-prototyping process.

Other methods to calculate the registration transformation without the need for extensive preoperative preparation utilize intraoperative imaging. As described above, a calibrated fluoroscope may be utilized to acquire VOs intraoperatively. Since the fluoroscope is tracked by the navigator during image acquisition and the relation between the fluoroscope's position in space and the resulting image is known, the twodimensional projective representations can be matched with a three-dimensional CT dataset yielding the registration of the preoperative scan. From a technical standpoint, such a procedure is nontrivial. One approach is to first extract bone contours from the fluoroscopic images, which can be a time-consuming interactive task [26]. Alternatively, the registration algorithm can be initiated with a coarse registration. Verheyden et al. [27] presented such an approach for spinal surgery. They had to acquire a pair of $\mathrm{C}$-arm images in a well-defined way and manually align them roughly with the preoperative CT. Nevertheless, their system did not prove to be reliable in every case.

Another alternative is the employment of intraoperative ultrasonography. If an ultrasound probe is tracked by a navigator and its measurements are calibrated, it may serve as a space digitizer with which position data of the anatomy may be acquired. It can, thus, replace any other tracked instrument to digitize landmarks for paired-points or surface registration. An ultrasound probe operates in either one of two different modes. A-(amplitude-)mode ultrasound yields the perpendicular depth along the acoustic axis of the device. Placed cutaneously, it can measure the distance to tissue borders, and the resulting point coordinates can be processed by any registration algorithm. Although the applicability of this technique has been demonstrated $[28,29]$, it is not used widely. The nature of A-mode ultrasound requires the probe to be oriented perpendicularly to the bone surfaces at which it is aimed. Moreover, the velocity of sound varies depending on the properties of the tissues that are traversed, thus leading to unpredictable inaccuracies when used to digitize deeply located structures. As a consequence, the successful use of this technique remains limited to a narrow 
field of application [30]. In contrast to the A-mode method, B-(brightness-)mode ultrasound scans a fanshaped area. It is therefore able to detect also surfaces that are examined from an oblique direction. In order to extract the relevant information for the registration of preoperative CT scans, the resulting, usually noisy images need to be processed either manually [31] or automatically [32]. As for the intraoperative processing of fluoroscopic images, the use of B-mode ultrasound for registration is not reliable in every case and is, consequently, a subject of continuous research.

If any intraoperative method is used to generate the $\mathrm{VO}$, registration is an inherent process. As stated above, the imaging device is tracked during data acquisition. As a result, the position of the acquired image is known with respect to the TO. This relation corresponds to the interactive registration in the case of preoperative images serving as VOs. Therefore, registration is not an issue when using intraoperative CT, two- or threedimensional fluoroscopy, or the SDA concept.

\section{Navigator}

Registration closes the gap between VO and TO. The navigator enables this connection by providing a global coordinate space. In addition, it links the surgical instruments, with which a procedure is carried out, to the TO that they act upon. From a theoretical standpoint, it is the only element in which surgical navigation systems and surgical robotic systems differ.

\section{Robots}

For surgical robotic systems, the robot itself is the navigator. It is registered to the $\mathrm{VO}$ which enables it to realize the plan that was defined by the surgeon in the preoperative image. Its actuators carry out specific tasks as part of the therapeutic treatment. Active robots act directly on the patient. They perform a specific task autonomously without additional support by the surgeon. Two robotic systems for total joint replacement have been developed $[33,34]$, but their clinical benefit has been strongly questioned lately $[23,35,36]$. Moreover, they require considerable investments while serving merely a rather limited field of applications. As a result, the future of these devices is highly uncertain. For traumatology applications, the use of robots has only been explored in laboratory settings so far [37]. This may be attributable to the nature of fracture treatment, which is usually a process that needs to be individualized for each case and seldom includes many standardized steps that a robot could repetitively carry out. Nevertheless, a robotic system for the reduction of long bone fractures has been realized [38]. It can be described as motorized Ilizarov external fixators, with which a planned motion path of a fragment can be reduced automatically. However, the device has a potentially larger field of application in corrective surgery, e.g., during callus distraction. Thanks to its computer-controlled interface, the normally difficult control of the motion of the parallel platforms is made easier, and continuous micromotions can be realized over a long period of time.

In contrast to these active robotic devices, semiactive robots do not carry out a part of the intervention autonomously, but rather guide or assist the surgeon who performs the operative action. At present, there are two representatives of this class that are commercially available, both for bone resection during total knee replacement. The Acrobot system [39] is based on an industrial robot, similar to the aforementioned machines for total hip replacement. It differs, however, in the purpose that it serves intraoperatively. It holds a high-speed mill that the surgeon is allowed to move freely in order to resect bone as long as this motion stays within a preoperatively defined safety volume. When the milling action is about to leave this volume causing more tissue to be resected than planned, the robot would actively intercept to block the unwanted movements. This approach enables the surgeon to carry out the actual resection process manually while being assured that the planned cuts are realized precisely. Other semi-active robots can be seen as intelligent gauges that place, e.g., cutting jigs or drilling guides automatically. Prototypes of this kind of machines have been presented [40, 41], and one commercially available product exists. This Galileo system [42] is a microrobot that is attached to the femur during total knee replacement. It aligns conventional cutting jigs in order to enable accurate preparation for the implant.

\section{Trackers}

The navigator of a surgical navigation system is a tracker. This measurement device remotely determines the position and orientation of objects and provides this data as three-dimensional coordinates. From a physical point of view, a number of methods exist to remotely sense the location of objects, and basically all of them have been implemented in trackers that, in turn, were used as parts of navigation systems. Most of today's products rely on optical tracking of objects using operating room-(OR-)compatible infrared light that is 
either actively emitted from the observed objects or passively reflected by them. In any case, a camera system registers these signals and reconstructs position data from them. To track surgical instruments with this technology requires the tools to be adapted with probes holding either light-emitting diodes (LED, active) or light-reflecting spheres or plates (passive, see Figure 6a). Depending on the tracker model used, the active LEDs are powered and controlled either by a cable or remotely, which requires a battery to be housed by the probe as well (Figure 6b). Tracking by means of video images has been suggested [43] as an inexpensive and simple alternative to a passive optical tracker, but the accuracy of this approach cannot compete with what infrared light-based systems can achieve.

Tracking using ultrasonic emitters and sensors has also been proposed [44]. Although this technique is well established for biomechanical motion analysis [45], it did not prove to be competitive for surgical navigation, because the achievable accuracy depends on the room temperature that influences the velocity of sound, thus requiring extensive calibration steps prior to each intraoperative use. Moreover, sterilization of the adopted surgical instruments is less easy than for optically tracked objects.

Both optical and acoustic tracking of surgical instruments requires a direct line of sight between the tracker and the observed objects. This can be a critical issue in OR setups where the camera or sonographic tracker needs free access to the situs. The use of electromagnetic tracking systems is believed to overcome this disadvantage. There, a homogeneous magnetic field is generated by an emitter coil. Receiver coils are attached to each of the instruments to be tracked that allow measuring their position and orientation within the magnetic field. This technique senses positions even if objects such as the surgeon's hand are in between the emitter coil and the tracked instrument. However, the homogeneity of the magnetic field can be easily disturbed by the presence of metal objects causing measurement artifacts that may decrease the achievable accuracy considerably [46]. Therefore, magnetic tracking is not employed in today's navigation systems anymore either, although it had been in clinical use in the past [47]. Probably one of the most obvious ways to track an instrument's position is by means of a direct mechanical link. Multilink arms have been known for many years to be reliable and precise measurement devices. It is obvious though, that the physical link between the arm and a small surgical instrument is not generally suitable due to the clumsiness of the devices. As a result, the field of application of mechanical trackers as parts of surgical navigation systems is narrow [48], and no clinical results are available.

\section{Referencing}

Relative motions between the TO and the navigator need to be detected and compensated. To do so, the operated anatomy is linked to the navigator. For robotic surgery, this connection is established as a physical linkage. Large active robots such as the machines used for total joint replacement, come with a bone clamp that tightly grips the treated structure, while the smaller semi-active devices are mounted directly onto the bone. They therefore compensate for relative motions by

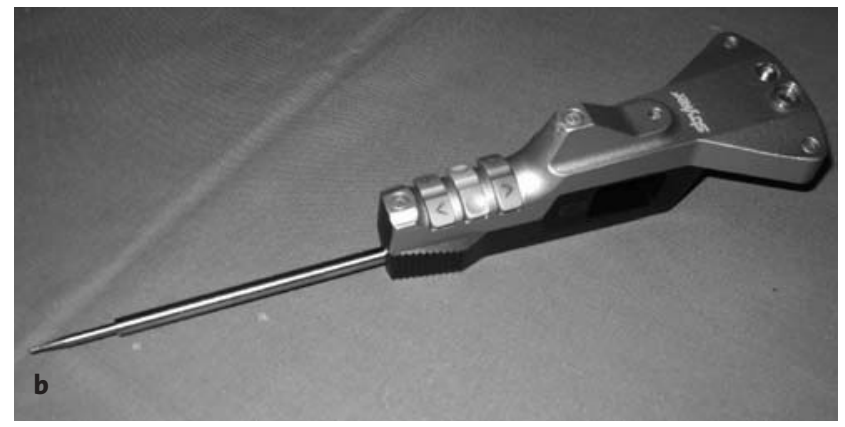

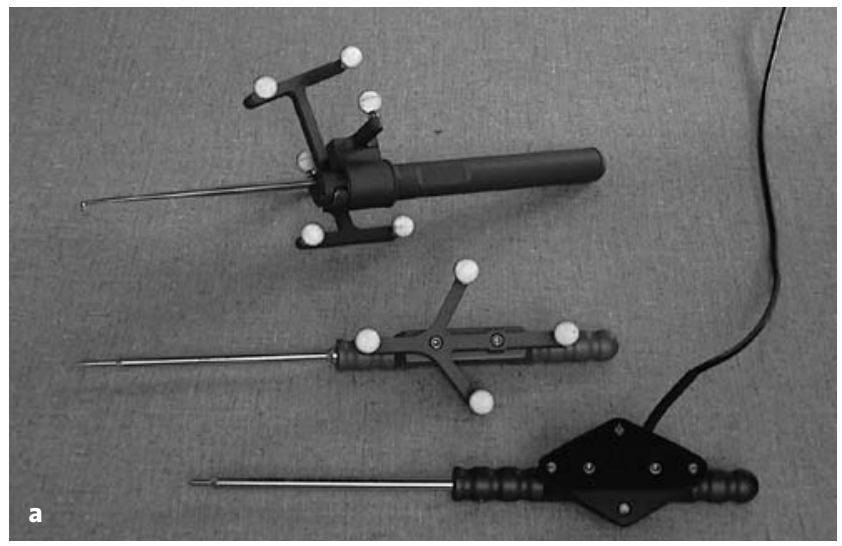

Figures $\mathbf{6 a}$ and $\mathbf{6 b}$. Optically tracked surgical instruments. a) Reflective spheres may be used for the passive tracking of instruments. These markers reflect infrared light that is emitted by the camera system. By contrast, most of the instruments that are tracked using actively light-emitting diodes are controlled and powered via cables that connect the instruments to the tracker. b) When active tracking of instruments shall be performed without cables, batteries and additional electronics need to be mounted to the instruments. 
eliminating them. An equivalent strategy is required when a mechanical arm is used as the navigator of a navigation system. For all other tracker types, bone motion is determined by the attachment of a so-called dynamic reference base (DRB) [49]. It houses infrared LEDs, reflecting markers, acoustic sensors, or an electromagnetic coil - depending of the employed tracking technology - and makes the TO visible to the tracker. Figure 7 shows the example of a DRB for an active optical tracking system that is attached to the spinous process of a lumbar vertebra. Since the DRB is used as an indicator to inform the tracker precisely about movements of the operated bone, stable fixation throughout the entire duration of the navigated procedure is essential.

\section{Clinical Fields of Application}

Since the mid-1990s when the first CAOS systems were successfully applied during total hip replacement and the insertion of pedicle screws into the lumbar spine, a large number of systems covering a wide range of traumatologic and orthopedic operations have been developed, clinically tested, and partly abandoned again, because the anticipated benefits failed to be achieved or the technology proved to be unreliable or too complex to be used intraoperatively. Discussing all these approaches and methods would go beyond the focus of this article. Nevertheless, a review of the most important systems and the most original technological approaches shall be presented here.

While there was clearly one pioneering example of robot-assisted orthopedic surgery, ROBODOC [33], several research groups first developed spinal navigation systems independently from each other, yet almost in parallel [47, 49-53]. These systems used preoperative CT scans for VOs, relied upon paired points and surface matching for registration, and used different optical or electromagnetic trackers. Their clinical success [54-56] made them initiate a worldwide search for further applications and boosted the development of new CAOS systems. While some groups tried to use the existing pedicle screw placement systems for other clinical applications [57-60], others aimed to apply the underlying technical principle to new clinical challenges by developing highly specialized navigation systems [24, 61, 62]. With the advent of alternative imaging methods for the generation of VOs, the indication for the use of one or the other method was evaluated more critically. For instance, it became evident that lumbar pedicle screw insertion in the standard degenerative case could be car-

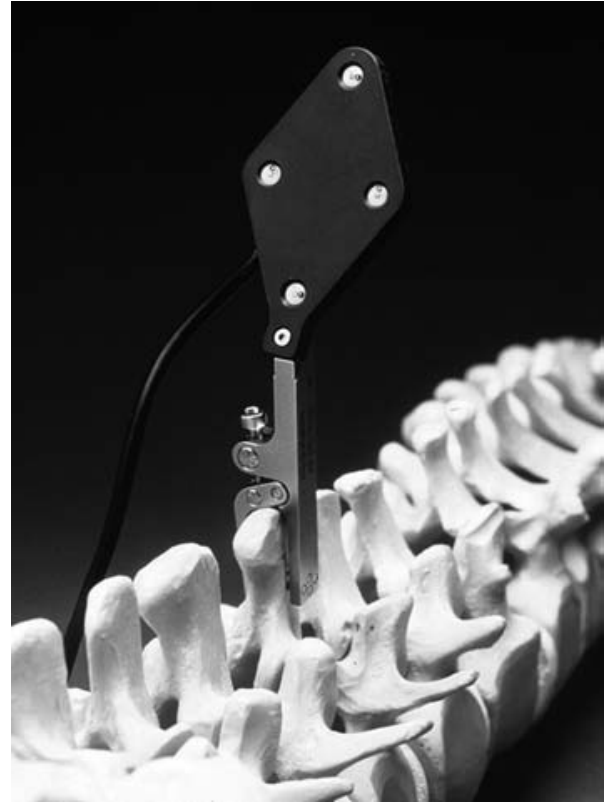

Figure 7. Dynamic reference base (DRB). A DRB allows a navigation system to track the anatomic structure on which the surgeon is operating. In the case of spinal surgery, this DRB is usually attached to the spinous process with the help of a clamping mechanism. It is essential that it remains rigidly affixed during the entire usage of the navigation system on that vertebra.

ried out with fluoroscopy-based navigation sufficiently accurately; it does usually not require the use of the more expensive CT-based method.

A comparable development could be observed for total knee replacement. Initially, this procedure was supported by active [63] and semi-active [39] robots, as well as navigation systems using preoperative CTs [64] or the SDA approach [65-67]. It is nowadays commonly accepted that neither active robotic devices nor navigation systems using preoperative images lead to optimal results.

Fluoroscopy-based navigation still seems to have a large potential to explore new fields of application. Up to now, mainly spinal surgery $[13,68]$ and total hip replacement [69] have been the focus of this technique. Although some efforts have been made to apply fluoronavigation to the treatment of long bone fractures [70], it is not widely used yet. In particular, the use of fluoro$\mathrm{CT}$ as a virtual object generator is currently being explored intensively [71, 72], mainly because of the versatility of this intraoperative imaging device.

\section{Conclusion}

Some 10 years after the first robots and navigation systems conquered the surgical theaters of orthopedic and 
trauma clinics, these technologies are still undergoing a rapid process of evolution. New techniques are invented or derived from existing methods; they are evaluated clinically and then modified, abandoned, or become a quasi-standard in a small number of centers. Up to now, none of the CAOS methods has succeeded in becoming state-of-the-art within the broader medical community. However, there seem to be a number of applications for which more and more studies prove the superiority of the CAOS approaches over classic techniques. It may be hypothesized that ultimate acceptance of robotic or navigated bone surgery will depend on furnishing proof of better long-term results. Consequently, more prospective and retrospective studies comparing the outcome of CAOS versus non-CAOS procedures with long followup times will have to be conducted.

\section{Acknowledgments}

Parts of this work were funded by the M.E. Müller Foundation, Bern, Switzerland, the AO/ASIF Foundation, Davos, Switzerland, and the Swiss National Science Foundation through the National Center of Competence in Research CO-ME, Zurich, Switzerland.

\section{References}

1. D'Lima DD, Urquhart AG, Buehler KO, et al. The effect of the orientation of the acetabular and femoral components on the range of motion of the hip at different head-neck ratios. J Bone Joint Surg Am 2000;82:315-21.

2. George DC, Krag MH, Johnson CC, et al. Hole preparation techniques for transpedicle screws - effect on pull-out strength from human cadaveric vertebrae. Spine 1991;16:181-4.

3. Davne SH, Myers DL. Complications of lumbar spinal fusion with transpedicular instrumentation. Spine 1992;17:S184-8.

4. Digioia AM 3rd, Jaramaz B, Plakseychuk AY, et al. Comparison of a mechanical acetabular alignment guide with computer placement of the socket. J Arthroplasty 2002;17:359-64.

5. Langlotz F. State-of-the-art in orthopaedic surgical navigation with a focus on medical image modalities. J Vis Comput Animat 2002;13:77-83.

6. Bowersox JC, Bucholz RD, Delp SL, et al. Excerpts from the final report for the Second International Workshop on Robotics and Computer Assisted Medical Interventions, Bristol, England, June 23-26, 1996. Comput Aided Surg 1997;22:69-101.

7. Martel AL, Heid O, Slomczykowski M, Kerslake R, Nolte LP. Assessment of 3-dimensional magnetic resonance imaging fast low angle shot images for computer assisted spinal surgery. Comput Aided Surg 1998;3:40-4.

8. Suhm N, Simeria A, Hügli R, et al. MR-imaging of bone cements as a first step towards MR-guided vertebroplasty. In: Langlotz F, Davies BL, Bauer A, eds. Computer assisted orthopaedic surgery3rd Annual Meeting of CAOS-International (Proceedings). Darmstadt: Steinkopff, 2003:356-7.

9. Jacob AL, Messmer P, Kaim A, et al. A whole-body registrationfree navigation system for image-guided surgery and interventional radiology. Invest Radiol 2000;35:279-88.

10. Messmer P, Jacob AL, Fries E, et al. Technologieintegration und Prozessmanagement - Konzept und Implementierung einer neuartigen Plattform für einzeitige Diagnostik und Therapie des akut Kranken und Verletzten sowie für elektive computerassistierte Chirurgie (CAS). Unfallchirurg 2001;104:1025-30.

11. Hofstetter R, Slomczykowski M, Bourquin Y, et al. Fluoroscopy based surgical navigation: concept and clinical applications. In: Lemke HU, Vannier MW, Inamura K, eds. Computer assisted radiology and surgery. Amsterdam: Elsevier Science, 1997: 956-6o.

12. Joskowicz L, Milgrom C, Simkin A, et al. FRACAS: a system for computer-aided image-guided long bone fracture surgery. Comput Aided Surg 1998;36:271-88.

13. Foley KT, Simon DA, Rampersaud YR. Virtual fluoroscopy: computer-assisted fluoroscopic navigation. Spine 2001;26:347-51.

14. Heiland $M$, Schulze D, Adam G, et al. 3D-imaging of the facial skeleton with an isocentric mobile C-arm system (Siremobil IsoC3D). Dentomaxillofac Radiol 2003;321:21-5.

15. Sati $M$, Stäubli HU, Bourquin $Y$, et al. Real-time computerized in situ guidance system for ACL graft placement. Comput Aided Surg 2002;7:25-40.

16. Fleute $M$, Lavallée $S$, Julliard R. Incorporating a statistically based shape model into a system for computer assisted anterior cruciate ligament surgery. Med Imaging Anal 1999;3:209-22.

17. Stindel E, Briard JL, Merloz P, et al. Bone morphing: $3 \mathrm{D}$ morphological data for total knee arthroplasty. Comput Aided Surg 2002;7:156-68.

18. Lavallée S. Registration for computer-integrated surgery: methodology, start of the art. In: Taylor RH, Lavallée S, Burdea GC, et al., eds. Computer integrated surgery. Cambridge: MIT Press, 1996:77-97.

19. Bargar WL, Bauer A, Börner M. Primary and revision total hip replacement using the Robodoc system. Clin Orthop 1998;354:82-91.

20. Winkler D, Vitzthum HE, Seifert V. Spinal markers: a new method for increasing accuracy in spinal navigation. Comput Aided Surg 1999;4:101-4.

21. Langlotz F, Stucki M, Bächler R, et al. The first twelve cases of computer assisted periacetabular osteotomy. Comput Aided Surg 1997;2:317-26.

22. Lund $T$, Schwarzenbach $O$, Jost $B$, et al. On minimally invasive lumboscaral spinal stabilization. In: Nolte LP, Ganz E, eds. Computer assisted orthopedic surgery (CAOS). Seattle: Hogrefe \& Huber, 1999:114-20.

23. Nogler M, Maurer H, Wimmer C, et al. Knee pain caused by a fiducial marker in the medial femoral condyle: a clinical and anatomic study of 20 cases. Acta Orthop Scand 2001;72:477-80.

24. Radermacher K, Portheine F, Anton $M$, et al. Computer assisted orthopaedic surgery with image based individual templates. Clin Orthop 1998;354:28-38.

25. Valstar ER, van Brussel K, Kaptein BL, et al. CT-based personalized templates for accurate glenoid prosthesis placement in total shoulder arthroplasty. In: Langlotz F, Davies BL, Bauer A, eds. Computer assisted orthopaedic surgery - 3rd Annual Meeting of CAOS-International (Proceedings). Darmstadt: Steinkopff, 2003:380-1.

26. Hamadeh A, Lavallée S, Cinquin P. Automated 3-dimensional computed tomographic and fluoroscopic image registration. Comput Aided Surg 1998;31:11-9.

27. Verheyden AP, Glasmacher S, Hölzl A, et al. First experiences with CT-fluoromatching navigation of transoral $\mathrm{C}_{1}$ instrumentation in displaced atlas fractures using the ENT headset. In: Langlotz F, Davies BL, Bauer A, eds. Computer assisted orthopaedic surgery 3rd Annual Meeting of CAOS-International (Proceedings). Darmstadt: Steinkopff, 2003:386-7.

28. Maurer CR, Gaston RP, Hill DLG, et al. AcouStick: a tracked Amode ultrasonography system for registration in image-guided 
surgery. In: Taylor C, Colchester A, eds. Medical image computing and computer-assisted intervention - MICCAI '99. Berlin: Springer, 1999:953-62.

29. Hüfner T, Oszwald M, Kfuri M Jr, et al. A-mode ultrasound registration in computer assisted pelvic surgery. In: Langlotz F, Davies BL, Bauer A, eds. Computer assisted orthopaedic surgery - 3 rd Annual Meeting of CAOS-International (Proceedings). Darmstadt: Steinkopff, 2003:148-9.

30. Caversaccio M, Nolte LP, Häusler R. Present state and future perspectives of computer aided surgery in the field of ENT and skull base. Acta Otorhinolaryngol Belg 2002;56:51-9.

31. Tonetti J, Carrat L, Blendea S, et al. Clinical results of percutaneous pelvic surgery - computer assisted surgery using ultrasound compared to standard fluoroscopy. Comput Aided Surg 2001;64:204-11.

32. Amin DV, Kanade T, Digioia AM 3rd, et al. Ultrasound-based registration of the pelvic bone surface for surgical navigation. Comput Aided Surg 2001;6:48.

33. Mittelstadt B, Kazanzides P, Zuhars J, et al. The evolution of a surgical robot from prototype to human clinical use. In: Taylor RH, Lavallée S, Burdea GC, et al., eds. Computer integrated surgery. Cambridge: MIT Press, 1996:397-407.

34. Paul A. Operationsroboter in der Endoprothetik: Wie CASPAR Hand an die Hüfte legt. MMW Fortschr Med 1999;141:18.

35. Nogler M, Krismer M, Haid C, et al. Excessive heat generation during cutting of cement in the Robodoc hip-revision procedure. Acta Orthop Scand 2001;72:595-9.

36. Honl M, Dierk O, Gauck C, et al. Comparison of robotic-assisted and manual implantation of a primary total hip replacement. A prospective study. J Bone Joint Surg Am 2003;85:1470-8.

37. Kfuri M Jr, Gösling T, Westphal R, et al. Robotic assisted fracture reduction - application on femoral shaft. In: Langlotz F, Davies BL, Bauer A, eds. Computer assisted orthopaedic surgery-3rd Annual Meeting of CAOS-International (Proceedings). Darmstadt: Steinkopff, 2003:182-3.

38. Seide K, Wolter D. Computerassistierte Frakturreposition mit dem Hexapodfixateur externe. Trauma Berufskrankh 1999;1:127-30.

39. Jakopec M, Harris SJ, Rodriguez y Baena F, et al. The first clinical application of a "hands-on" robotic knee surgery system. Comput Aided Surg 2001;66:329-39.

40. Sautot $P$, Cinquin $P$, Lavallée $S$, et al. Computer assisted spine surgery: a first step toward clinical application in orthopaedics. In: Merucci JP, Plonsey R, Coatrieux JL, Laxminarayan S, eds. Proceedings of the 14th Annual Conference of the IEEE Engineering in Medicine and Biology Society. Piscataway: IEEE Inc.,: 1992: 1071-2.

41. Shoham M, Burman M, Zehavi E, et al. Bone mounted miniatur robot for surgical procedures - concept and applications. In: Langlotz F, Davies BL, Bauer A, eds. Computer assisted orthopaedic surgery - 3 rd Annual Meeting of CAOS-International (Proceedings). Darmstadt: Steinkopff, 2003:330-1.

42. Ritschl P, Machacek F, Fuiko R. Computer assisted ligament balancing in TKR using the Galileo system. In: Langlotz F, Davies BL, Bauer A, eds. Computer assisted orthopaedic surgery - 3rd Annual Meeting of CAOS-International (Proceedings). Darmstadt: Steinkopff, 2003:304-5.

43. De Siebenthal J, Langlotz F. Video tracking for intra-operative guidance - a complementary solution to tracking in CAS interventions. In: Langlotz F, Davies BL, Bauer A, eds. Computer assisted orthopaedic surgery - 3 rd Annual Meeting of CAOS-International (Proceedings). Darmstadt: Steinkopff, 2003:86-7.

44. Wallny T, Klose J, Steffny G, et al. Dreidimensionaler Ultraschall und intraoperative Navigation: ein neuer Einsatz des Ultraschall- topometers bei Umstellungsosteotomie des proximalen Femurs. Ultraschall Med 1999;20:158-60.

45. Huitema RB, Hof AL, Postema K. Ultrasonic motion analysis system - measurement of temporal and spatial gait parameters. J Biomech 2002;356:837-42.

46. Meskers CG, Fraterman H, van der Helm FC, et al. Calibration of the "Flock of Birds" electromagnetic tracking device and its application in shoulder motion studies. J Biomech 1999;32:629-33.

47. Amiot LP, Labelle H, Deguise JA, et al. Computer-assisted pedicle screw fixation. A feasibility study. Spine 1995;20:1208-12.

48. Mac-Thiong JM, Aubin CE, Dansereau J, et al. Registration and geometric modelling of the spine during scoliosis surgery: a comparison study of different pre-operative reconstruction techniques and intra-operative tracking systems. Med Biol Eng Comput 1999;37:445-50.

49. Nolte LP, Visarius H, Arm E, et al. Computer-aided fixation of spinal implants. J Image Guid Surg 1995;1:88-93.

50. Foley KT, Smith MM. Image-guided spine surgery. Neurosurg Clin N Am 1996;7:171-86.

51. Glossop ND, Hu RW, Randle JA. Computer-aided pedicle screw placement using frameless stereotaxis. Spine 1996;21: 2026-34.

52. Kalfas IH, Kormos DW, Murphy MA, et al. Application of frameless stereotaxy to pedicle screw fixation of the spine. J Neurosurg 1995;83:641-7.

53. Merloz P, Tonetti J, Pittet $L$, et al. Pedicle screw placement using image guided techniques. Clin Orthop 1998;354:39-48.

54. Amiot LP, Lang K, Putzier $M$, et al. Comparative results between conventional and computer-assisted pedicle screw installation in the thoracic, lumbar, and sacral spine. Spine 2000;25:606-14.

55. Laine $T$, Lund $T$, Ylikoski $M$, et al. Accuracy of pedicle screw insertion with and without computer assistance: a randomised controlled clinical study in 100 consecutive patients. Eur Spine J 2000;9:235-40.

56. Schwarzenbach O, Berlemann U, Jost B, et al. Accuracy of computer-assisted pedicle screw placement. An in vivo computed tomography analysis. Spine 1997;22:452-8.

57. Bale RJ, Fink C, Rosenberger R, et al. First experiences with computer assisted drilling of osteochondral lesions of the talus - feasibility and accuracy. In: Lemke HU, Vannier MW, Inamura K, et al., eds. Computer assisted radiology and surgery. Amsterdam: Elsevier Science, 1997:762-6.

58. Hoffer Z, Varga PP, Papik K. Computer-assisted percutaneous hip fixation - a case report. In: Lemke HU, Vannier MW, Inamura K, et al., eds. Computer assisted radiology and surgery. Amsterdam: Elsevier Science, 1997:1036.

59. Josten C, Hölzl A, Glasmacher S, et al. First experience with navigation of the pelvis. In: Langlotz F, Davies BL, Bauer A, eds. Computer assisted orthopaedic surgery - zrd Annual Meeting of CAOS-International (Proceedings). Darmstadt: Steinkopff, 2003:168-9.

6o. Leung KS, Yung SH, Tsui HF, et al. 3-D fluoro-navigation for articular fractures - a report of early experience. In: Langlotz F, Davies $\mathrm{BL}$, Bauer A, eds. Computer assisted orthopaedic surgery-3rd Annual Meeting of CAOS-International (Proceedings). Darmstadt: Steinkopff, 2003:204-5.

61. Digioia AM 3rd, Simon DA, Jaramaz B, et al. HipNav: preoperative planning and intraoperative navigational guidance for acetabular implant placement in total hip replacement surgery. In: Nolte LP, Ganz E, eds. Computer assisted orthopedic surgery (CAOS). Seattle: Hogrefe \& Huber, 1999:134-40.

62. Croitoru H, Ellis RE, Prihar R, et al. Fixation based surgery: a new technique for distal radius osteotomy. Comput Aided Surg 2001;6:160-9. 
63. Siebert W, Mai S, Kober R, et al. Technique and first clinical results of robot-assisted total knee replacement. Knee 2002;9:173-80.

64. Delp SL, Stulberg SD, Davies B, et al. Computer assisted knee replacement. Clin Orthop 1998;354:49-56.

65. Dessenne V, Lavallée S, Julliard R, et al. Computer assisted knee anterior cruciate ligament reconstruction: first clinical tests. J Image Guid Surg 1995;1:59-64.

66. Klos TVS, Habets RJE, Banks AZ, et al. Computer assistance in arthroscopic anterior cruciate ligament reconstruction. Clin Orthop 1998;354:65-9.

67. Krackow KA, Bayers-Thering M, Phillips MJ, et al. A new technique for determining proper mechanical axis alignment during total knee arthroplasty: progress toward computer-assisted TKA. Orthopedics 1999;22:698-702.

68. Nolte LP, Slomczykowksi MA, Berlemann U, et al. A new approach to computer-aided spine surgery: fluoroscopy-based surgical navigation. Eur Spine J 2000;9:S78-88.

69. Zheng G, Marx A, Langlotz U, et al. A hybrid CT-free navigation system for total hip arthroplasty. Comput Aided Surg 2002;7:129-45.

70. Suhm N, Jacob AL, Nolte LP, et al. Surgical navigation based on fluoroscopy - clinical application for computer-assisted distal locking of intramedullary implants. Comput Aided Surg 2000;5:391-400.
71. Fritsch E, Duchow J. Placement of pedicle screws at the entire spine with a new (Iso-C3D fluoroscopy) guiding system. In: Langlotz F, Davies BL, Bauer A, eds. Computer assisted orthopaedic surgery - 3 rd Annual Meeting of CAOS-International (Proceedings). Darmstadt: Steinkopff, 2003:106-7.

72. Grützner PA, Wälti H, Vock B, et al. Inherent spinal navigation using fluoro-CT technology. In: Langlotz F, Davies BL, Bauer A, eds. Computer assisted orthopaedic surgery - 3rd Annual Meeting of CAOS-International (Proceedings). Darmstadt: Steinkopff, 2003:128-9.

\section{Address for Correspondence}

Frank Langlotz, PhD

Institute for Surgical Technology and Biomechanics

M.E. Müller Research Center for Orthopedic Surgery University of Bern

P.O. Box 8354

3001 Bern

Switzerland

Phone (+41/31) 632-8679, Fax -4951

e-mail: Frank.Langlotz@MEMcenter.unibe.ch 